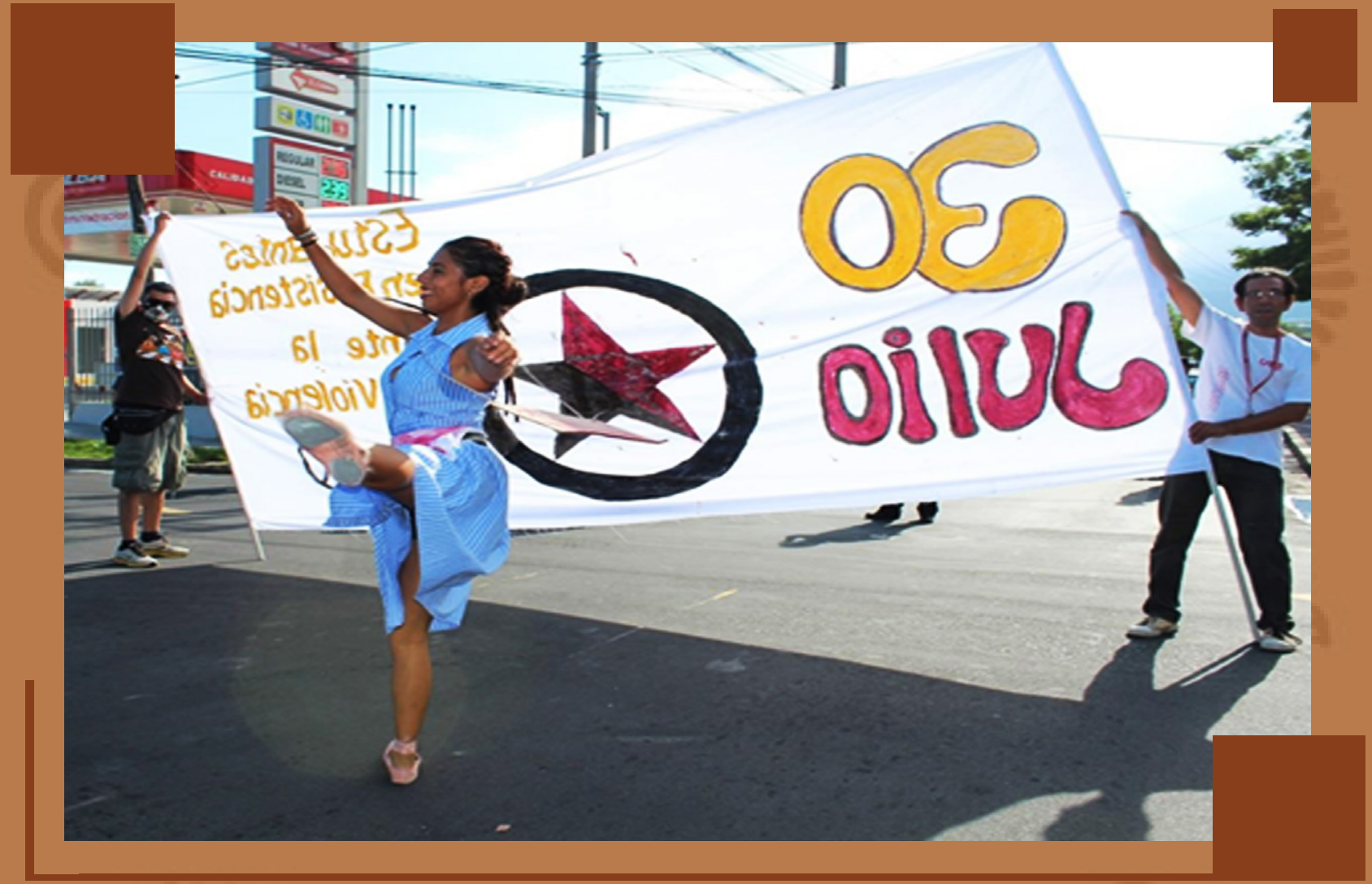

Apuntes metodológicos sobre la antropología de la acción pública

Estudios Interdiciplinarios 


\section{Apuntes metodológicos sobre la antropología de la acción pública}

\section{Methodological notes on the anthropology of public action}

\author{
Oscar Roberto Magaña Rivera \\ Universidad de El Salvador \\ ID Orcid: https://orcid.org/0000-0001-7061-7477 \\ oscar.mr94@gmail.com
}

\author{
Gloria Ester Peña Rodríguez \\ Universidad de El Salvador \\ ID Orcid: https://orcid.org/0000-0002-8137-7189 \\ gloriaester89@hotmail.com
}

Recibido: 27-10-2019

Aceptado: 20-11-2019

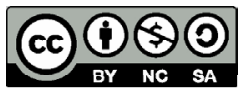

Copyright $\odot 2020$ UNAN-Managua Todos los Derechos Reservados.

\section{Resumen}

El estudio de las políticas públicas ha sido tradicionalmente un campo de estudio de las ciencias políticas. Usando como punto de partida la propuesta teórico-metodológica de la antropología de la acción pública propuesta por Rodríguez Castillo, Por la cual, las políticas públicas pasan a ser entendidas como un objeto de cultura, se abre un nuevo campo fértil para entrar a esta temática partiendo de estudios de carácter antropológico. La propuesta de este artículo desarrolla la investigación realizada sobre el aparato de Seguridad Pública en El Salvador y otra sobre la Política Cultural de la Universidad de El Salvador. Este enfoque posicionó a los investigadores en una realidad etnográfica diferente que llevó necesariamente a la aplicación de una metodología específica para el estudio de la acción pública.

Palabras claves: antropología, acción, políticas, públicas, etnografía

\section{Abstract}

The study of public policies has traditionally been a field of study of political science. Using as a starting point the theoretical-methodological proposal of the anthropology of public action proposed by Rodríguez Castillo, in which public policies become understood as an object of culture, a new fertile field is opened to enter this theme starting of anthropological studies. Based on this proposal, an investigation has been carried out on the Public Security apparatus in El Salvador and another on the Cultural Policy of the University of El Salvador. This approach positioned researchers in a different ethnographic reality that necessarily led to the application of a specific methodology for the study of public action.

Keywords: anthropology, action, politics, public, ethnography

Portada: Conmemoración del 30 de Julio en El Salvador, un hecho histórico conocido como la masacre estudiantil del 30 julio de 1975.

Foto: Gloria Ester Peña, 2019 


\section{Introducción}

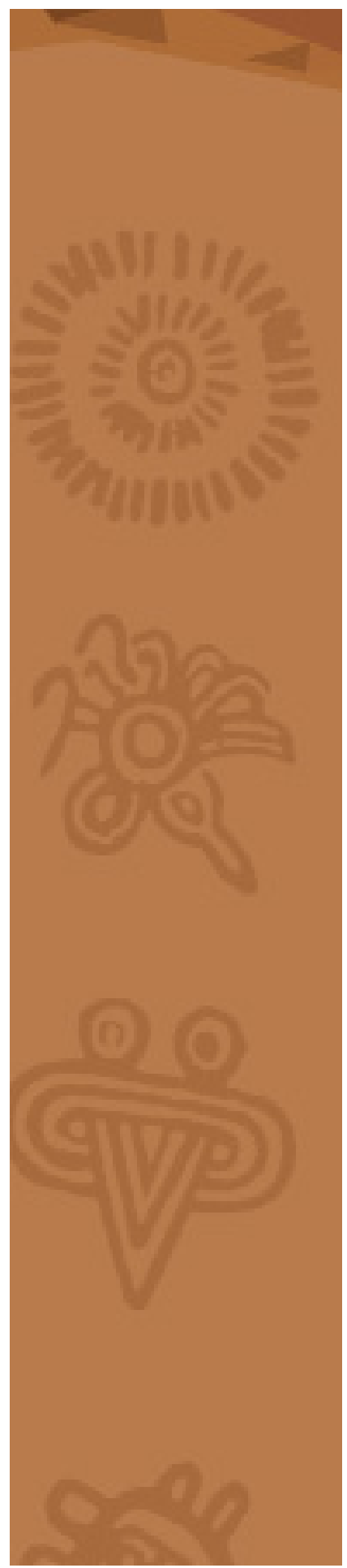

Este texto parte de los planteamientos teórico-metodológicos desarrollados en dos tesis de grado: "Análisis antropológico del proceso de políticas públicas de seguridad desde el aparato de seguridad pública" (Magaña, 2018) y "Antropología de la acción pública política cultural universitaria" (Peña, 2018). Ambas investigaciones parten desde la perspectiva de la antropología de la acción pública desarrollada por Rodríguez Castillo (2011), con el fin de estudiar las políticas de seguridad en el Estado y culturales en la Universidad de El Salvador respectivamente.

En primer lugar, se desarrolla brevemente qué es la antropología de la acción pública, su objeto de estudio y los conceptos en los que se fundamente. Luego, se pasa a explicar cómo esta propuesta sirve de base para desarrollar el estudio de las políticas partiendo de la noción de aparato, poniendo el énfasis en las relaciones y conexiones que se establecen, y cómo esto es útil para examinar el proceso de implementación de las políticas. Posteriormente, se explica el enfoque de los conflictos entre sujetos, agentes y agencias dentro del campo de las políticas, y cómo se contrastan los discursos con la acción pública, lo que determina y es determinado por los indicadores culturales en los sujetos involucrados.

El énfasis de este trabajo es mostrar cómo, partiendo de la propuesta de la acción pública, se construyeron los abordajes teórico-metodológicos que sirvieron para aproximarse a un contexto etnográfico diferente al tradicional en la ciencia antropológica: el de los agentes e instituciones partícipes del proceso de las políticas públicas.

\section{Antropología de la acción pública: una propuesta de estudio}

Se debe comprender que las políticas públicas no son un documento que rige el accionar de una institución, ni son el accionar mismo, sino que éstas se encuentran inmersas y trascendidas en un proceso complejo que "consiste en la identificación del problema público, la formulación de la agenda de gobierno, el diseño de políticas públicas, la implementación de programas y proyectos y, por último, la evaluación de los resultados" (Rodríguez, 2014, p. 166).

Tradicionalmente, las políticas públicas y su proceso han sido parte del campo de estudio de las ciencias políticas. Sin embargo, partiendo de entender a todo este proceso como una compleja maquinaria cuyos motores están siendo programados y manejados en diferentes escalas por varios tipos de operarios en todo momento de su funcionamiento, podremos entender que, quienes realmente dan forma y orientan el proceso es un complejo grupo de agentes sociales, cada uno con voluntad, medios, objetivos y metas propios pero que convergen en torno a un tema común: la política pública.

El ejemplo anterior nos lleva a concebir la política pública como un objeto de cultura, haciendo posible estudiarla desde la perspectiva de la antropología 
La gente históricamente ha sido vinculada a través de las conexiones sociales directas, caracterizadas por su tangibilidad, corporalidad y localidad social. Además, lleva a entender que, alrededor de las políticas públicas y su proceso, se genera una fuerte dinámica social entre agentes e instituciones policéntricas, denominada "acción pública intensa" (Rodríguez, 2011, p. 158).

Al hablar de cultura, se da la pauta para el abordaje antropológico, sin embargo, este término es el que más discusión y disenso genera dentro de nuestra disciplina, por lo que es necesario explicar la conceptualización de cultura de la que parte la presente perspectiva. Para esto, nos remitimos al concepto trabajado por Roberto Varela, para quien la cultura es el conjunto de "signos y símbolos que transmiten conocimientos e información, portan valores, suscitan emociones y sentimientos, expresan ilusiones y utopías" (Varela, 1997, p. 48).

Es así como todo este proceso se convierte en un espacio donde convergen una variedad de agentes policéntricos que poseen todo este conjunto de elementos e indicadores culturales, e impregnan de los mismos a las políticas públicas. Estudiar el proceso y a los agentes involucrados partiendo de dichos indicadores, es justamente la finalidad de la antropología de la acción pública propuesta por Luis Rodríguez (Rodríguez, 2011, p. 158).

\section{Antropología de la acción pública: los aparatos y la imple- mentación de las políticas}

\section{Relaciones, conexiones y aparatos: antropología en las instituciones}

Feldman propone dos formas de vinculación entre los agentes: las conexiones, que son interacciones que refieren a un vínculo directo, establecido mediante la convivencia física entre las personas; por su lado, las relaciones hacen referencia a las interacciones indirectas, generalmente mediadas por agencias, representaciones políticas, normas sociales, etc.

“¿Por qué la preferencia por las conexiones sobre las relaciones y qué son sus consecuencias? La pregunta es contestada mejor por recordarnos la diferencia entre ellos, aunque a menudo se mezclan juntos. La diferencia es principalmente que las conexiones implican contacto directo e inmediato entre la gente mientras las relaciones involucran contacto indirecto, mediado. La gente históricamente ha sido vinculada a través de las conexiones sociales directas, caracterizadas por su tangibilidad, corporalidad y localidad: el intercambio de bienes tangibles; rituales y ritos que implican contacto corporal y social; actos visibles de reverencia a los ancianos; y la tortura pública como una exhibición espectacular del antiguo poder soberano." (Feldman, 2011, p. 379)

La antropología se ha caracterizado por darle mayor relevancia a las conexiones por encima de las relaciones, ya que son se han encargado de vincular a las personas entre sí, además de ser fácilmente observables y ubicables en el espacio y tiempo, por lo que son mucho más susceptibles de ser estudiadas bajo la observación participante. 


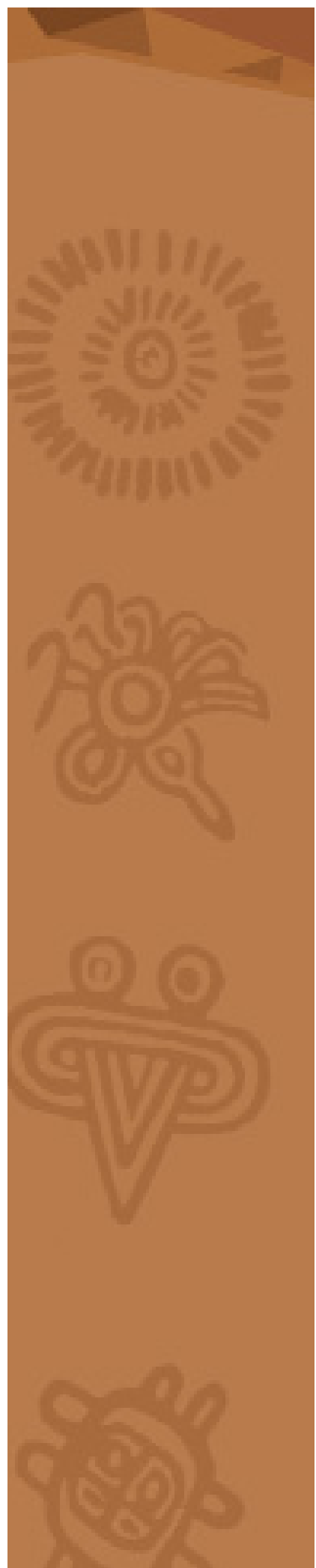

Las relaciones, por su parte, entrelazan agentes sin conexiones entre sí, por lo que no son ubicables en el espacio-tiempo, se forman de manera más sutiles, a través de agentes mediadores y, por tanto, la observación participante no puede dar cuenta de ellas a profundidad, surgiendo la necesidad de buscar un enfoque metodológico apropiado para dar cuenta de estas relaciones.

El entramado de estas relaciones que une a agentes diversos sin conexiones directas en una red social más amplia es lo que conforma los aparatos. Para definir "aparato", hay que remitirse a la noción establecida por Feldman a partir de los planteamientos de Rabinow, Foucault y DeGenova:

"[...] el aparato como un dispositivo de control de la población y gestión económica compuesta de elementos dispares y contrarios. Estos elementos unen en particular coyunturas históricas usualmente identificadas como "crisis" [...] El aparato es esencialmente la red que une a estos elementos [...] La identificación de "crisis" es, por tanto, la condición de posibilidad para un aparato (Feldman, 2011, p. 380).

Esta definición, recuerda inevitablemente al momento de "crisis" en el que un problema de gran relevancia para una población salta a la agenda pública, y una red deinstituciones diversas convergen en torno al trabajodeun tema en común.

Estamos hablando entonces del estudio del aparato como una forma de abordaje al estudio de las políticas públicas, poniendo el énfasis en las relaciones y conexiones entre los agentes y agencias involucrados. Se construye así un esbozo metodológico para el estudio de una política pública partiendo de la perspectiva de la antropología de la acción pública.

\section{Control, cambio y consenso: indicadores culturales en el proceso de las políticas públicas}

El concepto de control, entendido como asegurar que las reglas sean obedecidas y las órdenes cumplidas, se vuelve una categoría central en el estudio del proceso de las políticas (van Meter \& van Horn, 1993, p. 107).

Para poder observar cómo opera el control en las organizaciones y establecer comparaciones, es necesario entonces recurrir al concepto de acatamiento. Según los propios autores:

"Efectivamente, este elemento permite comparar muchas de las caracteristicas de las organizaciones complejas: las metas que persiguen, sus estructuras, sus mecanismos de motivación, el poder y la interacción que ejercen sus élites, el nivel y los tipos de consenso que logran y sus sistemas de comunicación y de socialización." (Van Meter \& van Horn, 1993, p. 108) 
Hay además dos elementos importantes para el éxito en la implementación de una política: el cambio y el consenso. El cambio se refiere por un lado a qué tanto varía una política de las que se han implementado anteriormente, y por el otro las reestructuraciones necesarias en las organizaciones responsables de implementarla.

El consenso remite a la aceptación y acuerdo entre los responsables de ordenar la implementación y quienes la implementan. Proponen, además que existe más resistencia a implementar una política que implique cambios drásticos que a la implementación de las políticas cuyos cambios sean graduales y progresivos. Además, será más difícil la implementación de políticas que gocen de poco consenso entre los agentes. Por esto, generalmente las políticas que implican alto grado de consenso o bajo grado de cambio tendrán una implementación más exitosa que aquellas con bajo consenso y cambios significativos. Dentro de esto, el nivel de consenso tiene mayor influencia que el elemento de cambio en la implementación. (Van Meter \& van Horn, 1993, p. 116).

Entre la política pública y su ejecución, existen varios elementos (variables independientes) que condicionan el proceso de implementación y determinan el grado de éxito en su ejecución (variable dependiente). Los elementos que van Meter y van Horn identifican son los estándares y objetivos, los recursos, la comunicación interorganizacional y actividades de inducción, las características de las agencias responsables de la implementación, las condiciones económicas, sociales y políticas, y la eficiencia de la implementación (van Meter \& van Horn, 1993).

\section{Antropología de la acción pública: abordaje de una política cultural}

\section{Poder y conflicto: arenas políticas en la antropología de la acción pública}

La sociedad presenta una aparente armonía dentro de sus alianzas, dilemas y contradicciones, Varela (1984) sostiene que lo político no se reduce a la competencia por el poder, sino que también es importante el análisis del conflicto; el conflicto ocupa un papel en los actores sociales (actores políticos) que contribuyen a la actividad política. Dentro del campo político en el que esta ocurre se observa una arena política: "una arena es un marco-institucionalizado o no-que manifiestamente funciona como un escenario para una interacción antagónica dirigida a llegar a una decisión públicamente reconocida" (Varela. 1984, p. 22). Una arena social consta de antagonismo que puede ser simbólico o factual, de un marco explícito y de una decisión publica que se puede llevar acabo con amenaza, persuasión o fuerza. Esto contribuye a las unidades desarmónicas (drama social). 


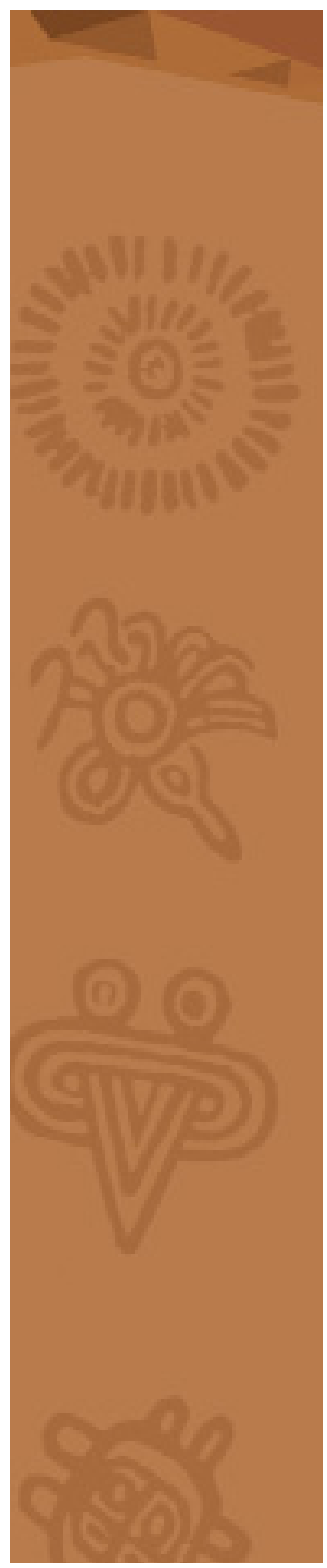

A su vez, se busca la propuesta de los contenidos culturales por los actores sociales tanto de prácticas políticas como de expectativas cotidianas en relación con las estructuras de poder (Domínguez. sf, p. 97) puesto que las "políticas públicas constituyen un ámbito político que interrelaciona a diversos agentes y grupos con intereses e identidades divergentes, los cuales se mueven en un amplio rango de espacios, tanto formales como informales" (Rodríguez Castillo en Domínguez. Sf, p. 102). Los espacios sociales generados por la acción pública configuran campos de disputa cultural donde se refuerzan o disputan relaciones de poder y dominación, entonces "la arena política, entendida como un espacio de condensación donde se enfrentan e interrelacionan los actores sociales" (Domínguez. Sf, p. 105).

El enfoque de acción pública intensa "debe permitirnos el análisis de los agentes sociales que están tanto a favor como en contra de una política pública" (Rodríguez; 2014:166) en este sentido es importante conocer lo que los agentes sociales dicen y hacen. Donde la base es la cultura, pero entendida en los términos de Varela (1997, p. 48).

\section{La mirada desde las instituciones hacia la cultura}

La antropología tiene como objetivo de estudio la cultura misma, la cual parte de un contexto social ya conocido por los sujetos pero que a la vez se va transformando. Esto es así desde la cotidianidad hasta los momentos en los cuales la cultura es normada, sistematizada, cualificada, cuantificada y politizada desde los paradigmas sociales, pues ya existen elementos de creatividad dentro del ser humano, y es cuando pasa por componentes de las estructuras sociales como las instituciones donde se encuentra con procesos normativos de control. Eagleton dice que "la naturaleza produce la cultura que a su vez transforma a la naturaleza" (Eagleton, 2001, p. 14).

Se dice, entonces, que existe un sentido dual entre lo natural y lo cultural, puesto que la cultura tiene un sentido de producción y desarrollo por su carácter transformador, pero a su vez es espontánea debido a su carácter natural pues lo que se transforma ya tiene su propia existencia autónoma. En tanto a las políticas culturales, hay que remitirse al artista como sujeto social, transformado y a la vez transformador de sus indicadores culturales. Él es libre, pero a la vez es sometido, creativo y condicionado y percibe esos elementos en términos de los otros, entonces se entiende que el individuo está bajo un marco regulador que sí permite una espontaneidad, pero bajo límites normativos a los que atañe la cultura.

En este sentido podemos entender que la cultura y el arte mismo parten de características inherentes al ser humano y que la expresión de estas en términos de practicidad y simbólicos se produce el surgimiento de discursos que responden a los intereses de los sujetos sociales.

Partiendo de lo anterior, se puede ver que el actuar del Estado contenido en la acción pública infunde a sus ciudadanos de disposiciones: 
Como ente regulador de las dimensiones de lo civil, entre la relación de Estado y ciudadano, queda la cultura, donde existe una pedagogía ética que prepara a la ciudadanía políticamente; de esa manera surge en el ciudadano la visión de un "yo" creado desde el Estado de tal manera que ha sido "cultivado y normado".

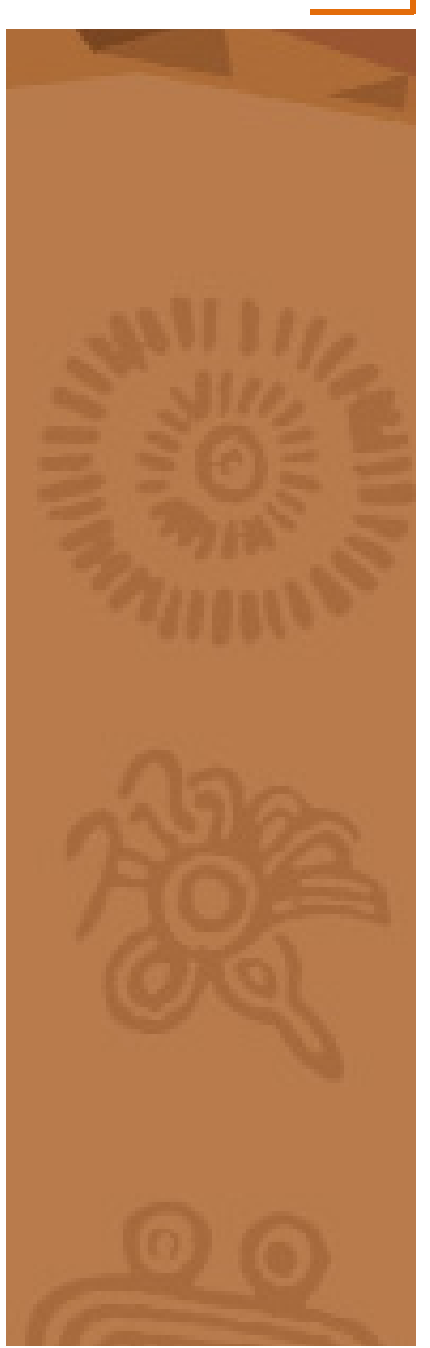

"En la sociedad civil los ciudadanos viven en un estado de antagonismo crónico, impulsado por intereses opuestos; pero el Estado es esa esfera transcendente en el que las divisiones se pueden reconciliar armónicamente. Sin embargo, para que ocurra esto, el Estado ya debe a ver ejercido su acción en la sociedad civil, aplacando los rencores y refinando las sensibilidades" (Eagleton. 2001, p. 14).

Como ente regulador de las dimensiones de lo civil, entre la relación de Estado y ciudadano, queda la cultura, donde existe una pedagogía ética que prepara a la ciudadanía políticamente; de esa manera surge en el ciudadano la visión de un "yo" creado desde el Estado de tal manera que ha sido "cultivado y normado".

Existen muchos planteamientos sobre las políticas culturales y su relación con el desarrollo cultural. Alejandro Grimson (2014, p. 10) plantea la cultura como un ente articulador para el desarrollo, ya que el actual sistema neoliberal dota de límites económicos y políticos al desarrollo de la cultura y de esta manera solo le atribuye dimensiones de aspecto decorativo limitando el accionar cultural. En países como los de Latinoamérica donde elementos del factor colonizador son muy fuertes, da la pauta para que el planteamiento neoliberal de la cultura sea impuesto y legitimado desde las instituciones reproduciendo el discurso homogeneizador de un Estado igualitario donde las características heterogeneidades responden a la lucha planteada desde las poblaciones subalternas.

Es así como el autor expone una desigualdad desde los grupos, entendiendo "nosotros y los otros". Así mismo las políticas neoliberales ven en las políticas culturales un gasto, donde según ven a la cultura como bienes culturales, tangibles e intangibles, expuestos como mercancía y de esta manera siguen promoviendo un incremento de los recursos.

Al efectuar cambios económicos y políticos se podría profundizar en las transformaciones culturales que llevarían al desarrollo cultural, pero no desde la perspectiva del desarrollo evolucionista del "nosotros, ellos" sino desde una perspectiva de un desarrollo articulatorio entre las instituciones y propuestas de políticas donde la cultura es el engranaje que permite la comprensión de todos los procesos de significación que mueven el accionar cultural de las personas. A consecuencia de esto se llevaría a cabo una lucha en contra de la exclusión y la desigualdad, y es ahí donde se observa el verdadero desarrollo cultural, entendiendo así que:

"Las políticas culturales, en un sentido amplio, son todas aquellas que pretenden incidir explícitamente en la configuración de procesos de significación, y el desarrollo cultural se refiere especificamente al proceso que incrementa la autonomía y libertad de los seres humanos, proceso que requiere a la vez bases materiales y simbólicas" (Grimson. 2014, pp. 10,11). 


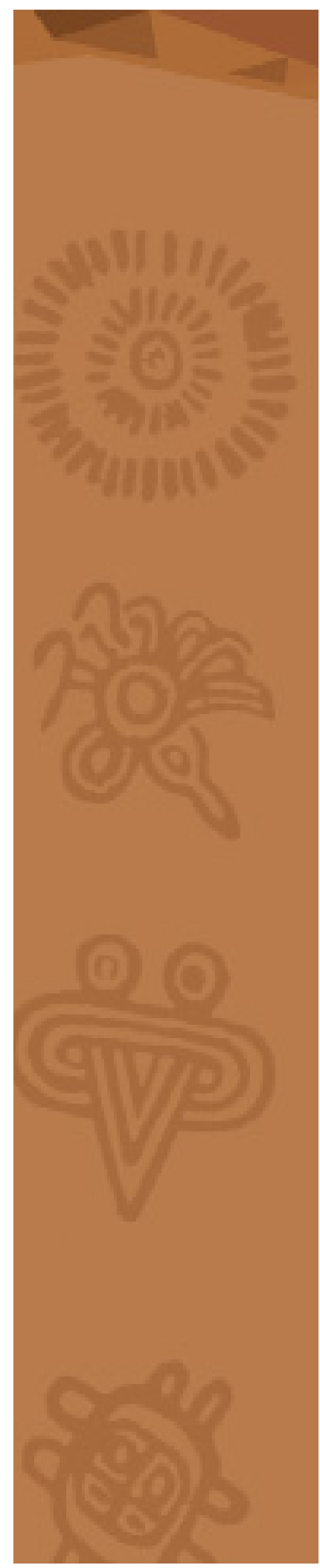

\section{Conclusión}

Las políticas públicas son el fruto de un complejo y dinámico proceso el cuál está siendo desarrollado en todo momento por sujetos sociales con un marco cultural, objetivos, intereses y perspectivas propias, divergentes entre sí pero que se aúnan en torno a un tema central: la política pública en símisma.

Si bien, este proceso ha sido estudiado desde las ciencias políticas y algunas ciencias sociales, el entendimiento de las políticas públicas como un objeto cultural permite realizar abordajes científicos partiendo de la ciencia antropológica. Esto es llamado antropología de la acción pública.

Esta propuesta es especialmente útil a la hora de realizar estudios de los procesos de políticas públicas que busquen dar cuenta de un problema de investigación partiendo del acercamiento con los sujetos sociales, la configuración de las redes de relaciones que se forman entre agencias y agentes, el contraste entre el discurso y la acción pública que se genera, es decir, lo que se dice y lo que se hace.

Ante esto es inevitable tomar en consideración que la etnografía como método por excelencia de la antropología también es cambiante y se transforma. Ésta debe adaptarse a la perspectiva utilizada, pero, sobre todo, a la realidad y contexto social que se pretende estudiar. Es así como la antropología de la acción pública lleva a desarrollar un marco teórico-metodológico específico, adaptado al tipo de política pública que se pretende estudiar y al proceso específico de ésta.

En este artículo se han contemplado dos casos concretos: el primero sobre cómo, partiendo de esta propuesta de estudio, se retoma la noción de aparatos, lo cual permite hacer un abordaje centrado en explicar las políticas públicas partiendo del estudio del entramado de redes que se tejen entre las relaciones y las conexiones entre agencias y agentes sociales.

El mapeo de esta configuración permite identificar los elementos que determinan mayormente a la política pública, y dar cuenta de cómo funcionan y se desarrollan las diferentes etapas de la misma.

El segundo caso es la aproximación, desde la antropología de la acción pública, a los conflictos y dramas sociales que se desarrollan en el campo de la política, retomando el concepto de arenas sociales. Dar cuenta de estos procesos de conflicto brinda otro crisol al investigador para observar el proceso de política y cómo los discursos en contraste con la acción social están determinando, e incluso imponiendo, un marco cultural en los sujetos involucrados. 


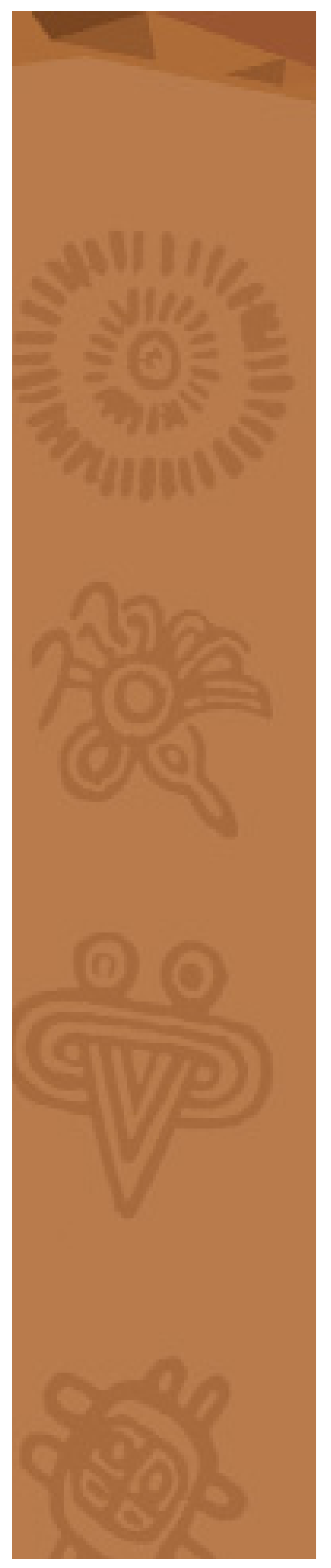

\section{Bibliografía}

Domínguez, E., Sf. Capítulo III, ¿Ciudadanía o Clientelismo? Paradojas de la Intermediación Política en las Arenas de la Política Social, pp. 96-199.

Eagleton, T., 2001. La Idea de Cultura, Barcelona, Ediciones Paidos Iberica.

Feldman, G., 2011. If ethnography is more than participant-observation, then relations are more than connections: The case for nonlocal ethnography in a world of apparatuses. Anthropological Theory, 11(4), pp. 375-395.

Grimson, A., 2013, Hegemonía Cultural y Políticas de la Diferencia, Ciudad Autónoma de Buenos Aires. Clacso.

Magaña, O., 2018. Análisis antropológico del proceso de políticas públicas de seguridad desde el aparato de seguridad pública. Bachelor thesis, Universidad de El Salvador.

Peña, G., 2018. Antropología de la acción pública política cultural universitaria. Bachelor thesis, Universidad de El Salvador.

Rodríguez, L., 2011. Hacia una etnografía de la acción pública: desafíos teórico-metodológicos de la antropología para el estudio de las políticas públicas contemporáneas. Maguaré, 25(1), pp. 131-163.

Rodríguez, L., 2014. Acción pública y desarrollo social en el sureste de México: la Agenda Chiapas-ONU. Liminar, XII(2), pp. 163-180.

van Meter, D. \& van Horn, C., 1993. El proceso de implementación de la') políticas. Un marco conceptual. En: L. Villanueva, ed. La implementación de las políticas. México D.F.: Miguel Ángel Porrúa, pp. 97-146.

Varela, R., 1997. Cultura y comportamiento. Alteridades, 7(13), pp. 47-52. 


\section{Oscar Roberto Magaña Rivera}

Graduado con honores de la Licenciatura en Antropología Sociocultural de la Universidad de El Salvador. Desarrolló su tesis de grado investigando sobre el proceso de las políticas públicas y el aparato de seguridad pública en El Salvador. Actualmente trabaja en el área de redacción y corrección editorial.

\section{Gloria Ester Peña Rodríguez}

Graduada de la Universidad de El Salvador de la carrera en Licenciatura en Antropología Sociocultural, desarrolló la investigación de grado en políticas públicas con el enfoque de Acción Publica Intensa, en el área de Arte y Cultura, en la ya mencionada universidad, analizando el abordaje de la institución hacia estos dos rubros y como el Estado mismo intervine en el desarrollo, visión, implementación y posicionamiento de la política en los sujetos de estudio; he realizado los siguientes diplomados en Debate Feminista, Diplomado en Economía Política, ambos impartido por la organización Mujeres Transformando, Diplomado en Historia de siglo XX, impartido por el Instituto Nacional de Formación Docente (INFOD), actualmente desarrollo el Diplomado en Educación y Democracia: Formación ciudadana para la escuela de hoy, impartido por, Saberes Docentes de la Universidad de Chile y el Instituto Nacional de Formación Docente (INFOD); trabajo y me desarrollo como docente en el área de Estudios Sociales, Lenguaje y literatura y materia de Moral, Urbana y Cívica (MUCi). 